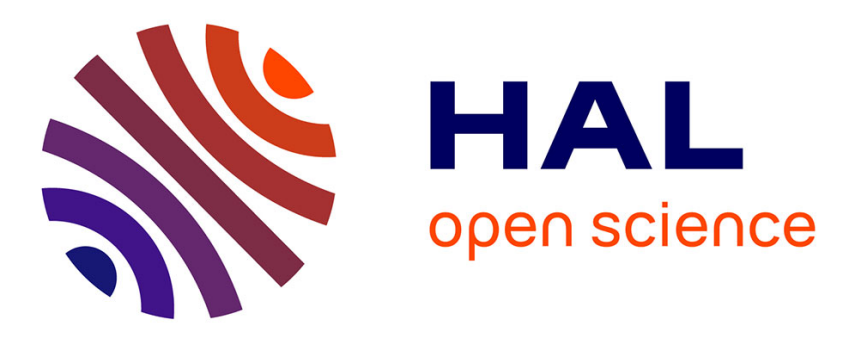

\title{
Time Resolved Correlation: a new tool for studying temporally heterogeneous dynamics
}

Luca Cipelletti, Hugo Bissig, Veronique Trappe, Pierre Ballesta, Sylvain Mazoyer

\section{- To cite this version:}

Luca Cipelletti, Hugo Bissig, Veronique Trappe, Pierre Ballesta, Sylvain Mazoyer. Time Resolved Correlation: a new tool for studying temporally heterogeneous dynamics. Journal of Physics: Condensed Matter, 2003, 15, pp.S257. hal-00004280

\section{HAL Id: hal-00004280 \\ https://hal.science/hal-00004280}

Submitted on 17 Feb 2005

HAL is a multi-disciplinary open access archive for the deposit and dissemination of scientific research documents, whether they are published or not. The documents may come from teaching and research institutions in France or abroad, or from public or private research centers.
L'archive ouverte pluridisciplinaire HAL, est destinée au dépôt et à la diffusion de documents scientifiques de niveau recherche, publiés ou non, émanant des établissements d'enseignement et de recherche français ou étrangers, des laboratoires publics ou privés. 


\title{
Time Resolved Correlation: a new tool for studying temporally heterogeneous dynamics
}

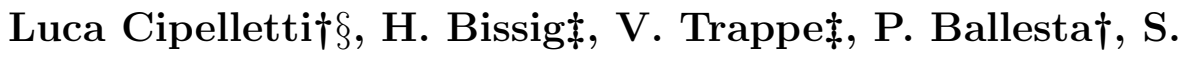 \\ Mazoyer $\dagger$ \\ $\dagger$ GDPC cc 26, Université Montpellier II, P. Battaillon 34095 Montpellier, France \\ $\ddagger$ University of Fribourg, Fribourg, Switzerland
}

\begin{abstract}
.
We introduce a new scheme to investigate temporally heterogeneous dynamics, which is termed Time Resolved Correlation (TRC). TRC is applied to data obtained by Diffusing Wave Spectroscopy probing the slow dynamics of a strongly aggregated colloidal gel. Other examples of TRC data, collected for different jammed materials in single and multiple scattering, are provided to demonstrate the wide range of applicability of this method. In all cases we find evidence that the slow dynamics results from a series of discrete steps rather than from a continuous motion, suggesting temporal heterogeneities to be a general feature of slow dynamics in jammed systems.
\end{abstract}

PACS numbers: $61.20 . \mathrm{Lc}, 82.70 . \mathrm{Dd}, 83.80 . \mathrm{Hj}, 78.35 .+\mathrm{c}$

Submitted to: J. Phys.: Condens. Matter

\section{Introduction}

Many soft materials, such as concentrated emulsions and suspensions, polymer glasses, foams, and gels are metastable, confined to a restrained region of configuration space. The mobility of their constituents is extremely reduced and they exhibit non-stationary, ultraslow dynamics that challenge both experimental characterization and theoretical description. Recent advances in scattering methods, such as the multispeckle technique [1], together with the development of conceptual tools, such as the jamming picture [2] and the concept of soft glassy materials [3], have provided new means of investigating these materials. However, the characterization of their dynamics and the understanding of its physical origin remain far from complete. In this paper, we describe a new approach to scattering techniques, which we call Time Resolved Correlation (TRC). This technique allows one to discriminate between continuous dynamics and intermittent or temporally heterogeneous dynamics. We present TRC measurements on concentrated colloidal gels and other jammed materials that suggest that temporal heterogeneities are

$\S$ To whom correspondence should be addressed (lucacip@gdpc.univ-montp2.fr) 

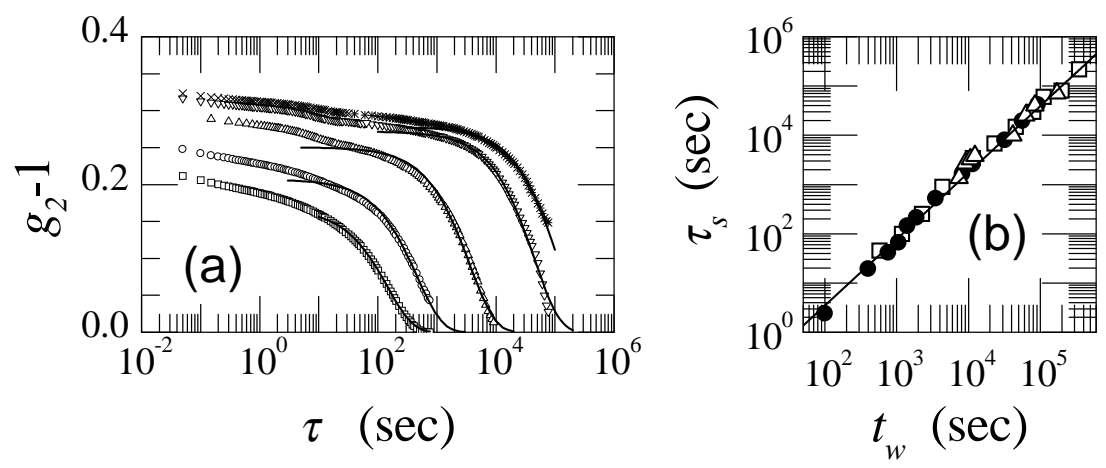

Figure 1. (a): $g_{2}-1$ measured by multispeckle DWS for a gel at $\phi=4.5 \%$. From bottom to top, $t_{w}$ is $0.6,1.1,4.0,31.2$, and 84 hours. (b): age dependence of the relaxation time of $g_{2}-1$. The different symbols refer to independent runs; the line is a power law fit to the data, yielding an exponent $\mu=1.31$.

a fundamental feature of the dynamics of jammed systems. These results highlight the potential of TRC as a powerful tool for gaining a better understanding of the dynamics of these systems.

\section{Slow dynamics of concentrated colloidal gels}

The colloidal gels are obtained by salt-induced aggregation of polystyrene particles of radius $a=85 \mathrm{~nm}$, suspended in a buoyancy-matching mixture of $\mathrm{H}_{2} \mathrm{O}$ and $\mathrm{D}_{2} \mathrm{O}$ at a volume fraction $\phi=4.5 \%$. The ions are produced in situ via an enzymatic reaction [4]. We take the time at which gelation occurs [5] as $t_{w}=0$, where $t_{w}$ is the sample age. Since the gels are very turbid, we probe their dynamics by Diffusing Wave Spectroscopy (DWS) [6], where the time autocorrelation function of the intensity of the light scattered under strong multiple scattering conditions is measured. In order to access the slow dynamics of the gels, we use the multispeckle technique [7]: the pixels of a charge coupled device (CCD) camera are used as detectors, and the correlation functions are averaged both over time and over several thousands of speckles, corresponding to the CCD pixels. With the multispeckle technique, the measurement time is of the order of the longest delay in the correlation function, several orders of magnitude less than with traditional techniques.

Figure 17a shows the intensity correlation functions $g_{2}(\tau)-1$ measured in the transmission geometry during the aging of the sample. Similarly to other jammed materials, $g_{2}(\tau)-1$ exhibits a fast decay (not accessible to the CCD, data not shown), followed by a slightly inclined plateau, before completely relaxing to zero. We focus on the final relaxation of $g_{2}(\tau)-1$ that can be described by an exponential decay, $\exp \left(-\tau / \tau_{s}\right)$, as shown by the fit (solid lines). The characteristic time $\tau_{s}$ increases with sample age as $t_{w}^{\mu}$, with $\mu=1.31$ (see (b)). Similar power-law behavior of the characteristic relaxation time measured by light scattering and rheology experiments has been observed for several jammed systems [1], although in most cases $\mu \leq 1$. By 
applying the standard DWS formalism [6], the particle mean squared displacement $<\Delta r^{2}(\tau)>$ can be calculated, yielding a diffusive-like behavior: $\left\langle\Delta r^{2}(\tau)>\sim \tau\right.$. The diffusion coefficient associated to this motion is age-dependent and varies between $2.9 \times 10^{-14} \mathrm{~cm}^{2} \mathrm{sec}^{-1}\left(t_{w}=100 \mathrm{sec}\right)$ and $5.6 \times 10^{-19} \mathrm{~cm}^{2} \mathrm{sec}^{-1}\left(t_{w}=4 \times 10^{5} \mathrm{sec}\right)$, many orders of magnitude smaller than that of the free particles $\left(2.5 \times 10^{-8} \mathrm{~cm}^{2} \mathrm{sec}^{-1}\right)$. Such an ultraslow diffusion of the constituents of a jammed system appears to be rather unphysical. Moreover, we note that single scattering experiments on similar gels, albeit at lower $\phi$, have ruled out diffusive motion as the physical mechanism responsible for the long time decay of the correlation functions [8]. A different approach is thus needed to account for the data shown in figure 11.

A slow exponential relaxation of $g_{2}(\tau)-1$ has been observed previously by Durian et al [9, 10] who investigated the dynamics in foams by DWS. They were able to demonstrate that this exponential relaxation is due to random rearrangements of the bubbles that are localized both in space and in time, rather than to the continuous motion of all scatterers, as is usually the case. Each rearrangement event completely randomizes the phase of the photons whose path crosses the rearranged region; accordingly, the decay time of the correlation function is the time it takes for a rearrangement event to occur along each photon path.

\section{Time Resolved Correlation (TRC)}

To test whether the dynamics of our gels, similarly to that of foams, is temporally heterogeneous we have developed a new scattering method that allows us to probe the evolution of the degree of correlation in time. We term this new approach Time Resolved Correlation (TRC); although TRC is very flexible and can be applied to single and multiple scattering experiments alike, we start by describing its implementation for the DWS experiments on the colloidal gels; an example of TRC data in the single scattering limit will be given at the end of the paper. We use the CCD camera of the multispeckle setup to take pictures of the speckle field scattered by the sample at regular time intervals (typically every 0.05 to $5 \mathrm{sec}$, depending on sample dynamics). The degree of correlation, $c_{I}\left(t_{w}, \tau\right)$, between pairs of images taken at a lag $\tau$ is then measured as a function of the time $t_{w}$ at which the first image was taken. The degree of correlation is calculated according to

$$
c_{I}\left(t_{w}, \tau\right)=\frac{<I_{p}\left(t_{w}\right) I_{p}\left(t_{w}+\tau\right)>_{p}}{<I_{p}\left(t_{w}\right)>_{p}<I_{p}\left(t_{w}+\tau\right)>_{p}}-1,
$$

where $I_{p}(t)$ is the intensity measured at time $t$ for the $p$-th CCD pixel and $\left\langle\cdots>_{p}\right.$ denotes the average over pixels. The quantity defined by (1) differs from the usual normalized intensity correlation function $g_{2}-1$ measured by traditional light scattering experiments in that the average is over pixels (associated to different speckles), rather than over time. Moreover, it differs from the correlation function calculated in multispeckle experiments, since in the latter case (11) is averaged either over time [7] (although less extensively than in single-detector experiments), or over several 

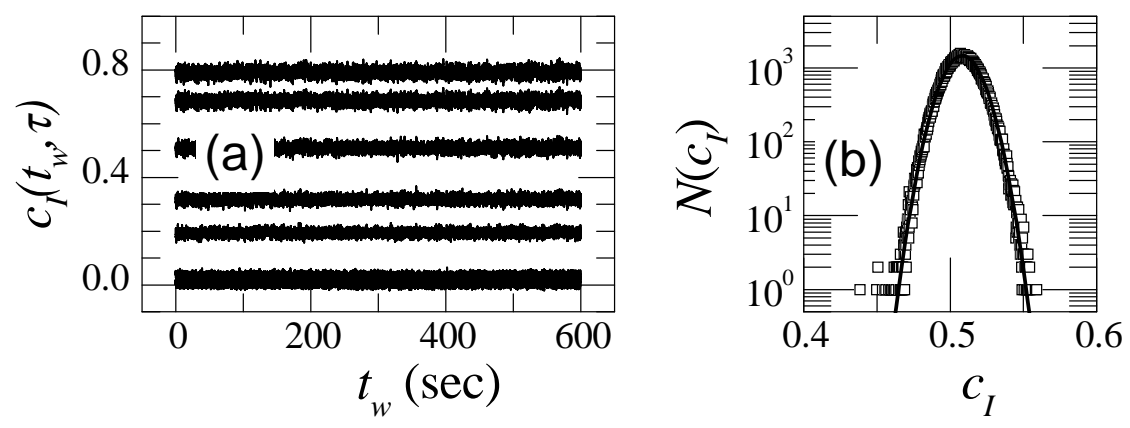

Figure 2. (a): TRC data for a brownian sample; from top to bottom, $\tau$ is $0.02,0.04$, $0.08,0.14,0.2,0.4,0.8,1.4$, and $2.0 \mathrm{sec}$. (b): frequency plot $N\left(c_{I}\right)$ of $c_{I}\left(t_{w}, \tau=0.08\right.$ sec) (squares). The line is a Gaussian fit to the data.

repetitions of the experiment [11] to obtain a true ensemble-averaged quantity. Finally, we observe that in usual scattering experiments $g_{2}(\tau)-1$ depends on the time lag $\tau$ as the independent variable, the sample age playing the role of a parameter should the dynamics be non-stationary. On the contrary, in a TRC measurement $\tau$ is taken as a parameter and a statistical analysis of $c_{I}\left(t_{w}, \tau\right)$ as a function of the independent variable $t_{w}$ is performed, thus allowing one to discriminate between continuous and temporally heterogeneous dynamics.

To demonstrate the method, we compare TRC data for a brownian system and for the gels. Figure 2a shows, for several lags $\tau$, the time evolution of $c_{I}\left(t_{w}, \tau\right)$ for a brownian sample, unaggregated polystyrene colloidal particles with radius $500 \mathrm{~nm}$ suspended in glycerol at a volume fraction $\phi=1.8 \%$. The data were collected in the same transmission geometry as for the gels. For a given $\tau, c_{I}\left(t_{w}, \tau\right)$ fluctuates randomly about a well defined mean value. Physically, this behavior indicates that the particles, on average, move by the same amount between any pairs of images, regardless of the time $t_{w}$ when the first image of the pair was taken, leading statistically to the same amount of loss of correlation of the scattered light. The fluctuations of $c_{I}\left(t_{w}, \tau\right)$ with $t_{w}$ are due solely to the measurement noise (finite number of speckles, CCD dark and readout noise): therefore, as a consequence of the Central Limit Theorem [12], we expect $c_{I}$ to be distributed as a Gaussian. This is indeed the case, as demonstrated by figure $2 \mathrm{~b}$, where a frequency plot of $c_{I}, N\left(c_{I}\right)$, is depicted on a semi-logarithmic scale for $\tau=0.08$ sec. The experimental points are obtained by grouping the data in intervals of $2 \times 10^{-4}$; the line is a Gaussian fit to the data, demonstrating the excellent agreement between $N\left(c_{I}\right)$ and a Gaussian. Although figure $2 \mathrm{~b}$ shows the frequency plot only for $\tau=0.08$ sec, we point out that similar results are obtained for all values of $\tau$. Note that the average over $t_{w}$ of $c_{I}\left(t_{w}, \tau\right)$ directly yields the intensity correlation function $g_{2}(\tau)-1$ as usually measured in a multispeckle experiment: the values of $c_{I}\left(t_{w}, \tau\right)$ are the outcome of single measurements of a quantity whose "true" value is estimated by $g_{2}(\tau)-1$. We find that $\left\langle c_{I}\left(t_{w}, \tau\right)\right\rangle_{t_{w}}=g_{2}(\tau)-1$ is very close to exponential, as predicted for brownian particles in the DWS transmission geometry. 

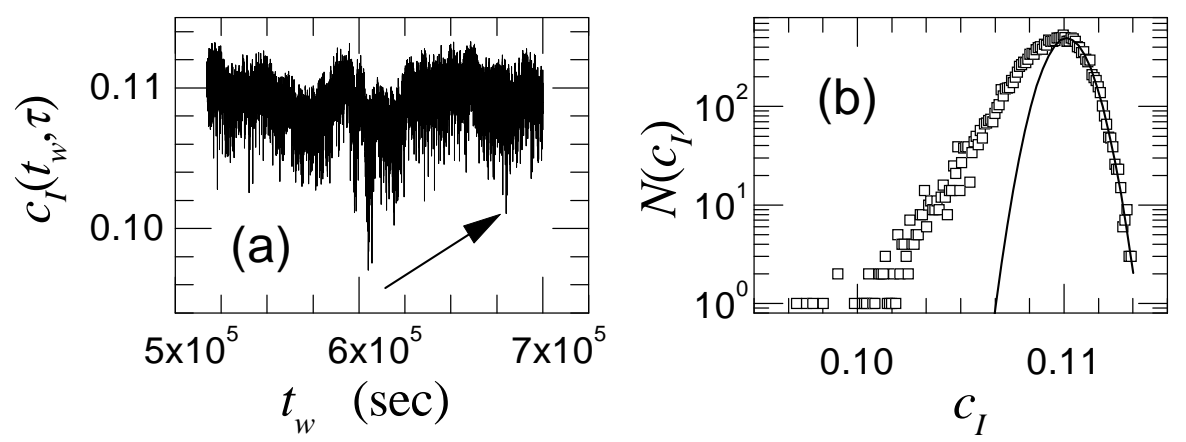

Figure 3. (a): TRC data for a colloidal gel at $\phi=4.5 \%$, for $\tau=2000$ sec. The arrow indicates one of the sudden, anomalously large loss of correlation. (b): frequency plot $N\left(c_{I}\right)$ of the data shown in (a). The line is a Gaussian obtained by fitting the data to the left of the maximum of $N\left(c_{I}\right)$.

Figure 4. NOTE: this figure may not show on cond-mat duer to its large size. Get the published version of this paper to see the figure. TRC data for a colloidal gel at $\phi=4.5 \%$, for $t_{w} \leq 15$ days. From top to bottom, $\tau$ is $10,100,1 \mathrm{k}, 2 \mathrm{k}, 4 \mathrm{k}, 10 \mathrm{~K}, 20 \mathrm{k}$, $40 \mathrm{k}, 100 \mathrm{k}$, and $200 \mathrm{k} \mathrm{sec}$.

Although the shape of the final decay of $g_{2}-1$ is the same for the colloidal gels as for the brownian sample, a totally different behavior is found for the TRC data. Figure 3a shows $c_{I}\left(t_{w}, \tau\right)$ for $\tau=2000 \mathrm{sec}$ and for $5.2 \times 10^{5}<t_{w}<7.0 \times 10^{5} \mathrm{sec}$, for the same sample as the one in figure 1. Contrary to the case of brownian particles, the noise of $c_{I}$ is not symmetric: remarkably, large drops of the degree of correlation can be clearly observed, such as the one pointed to by the arrow. These drops indicate that, from time to time, the particles undergo larger-than-usual movements: whenever such an anomalous motion occurs in between the time $t_{w}$ and $t_{w}+\tau$ at which two images of the speckle pattern are taken, the degree of correlation $c_{I}\left(t_{w}, \tau\right)$ between these two images exhibits a sudden drop. To better demonstrate the differences between the continuous dynamics of the brownian scatterers and the temporally heterogeneous dynamics of the gels, we show in figure $3 \mathrm{~b}$ the frequency plot for the TRC data of figure 3a. In sharp contrast to the case of figure $2 \mathrm{~b}$, the distribution $N\left(c_{I}\right)$ is non-Gaussian and is markedly skewed to the left: its skewness is due to rare events involving anomalously large movements that lead to a relevant loss of correlation. We remark that these events, localized in time and presumably in space, are strongly reminiscent of the "micro-rearrangements" introduced in a recent model for the dynamics of colloidal gels [13].

Figure 1 shows, for several $\tau$, the time evolution of $c_{I}\left(t_{w}, \tau\right)$ during 15 days. As a general trend, $c_{I}$ grows with $t_{w}$ so that, for a given $\tau$, the degree of correlation increases with sample age. This is equivalent to the increase of the characteristic relaxation time $\tau_{s}$ of $g_{2}-1$ already observed in figure 1, since the correlation functions become larger than zero for increasingly large delays $\tau$ as the sample ages. We note that at large $t_{w}$ 

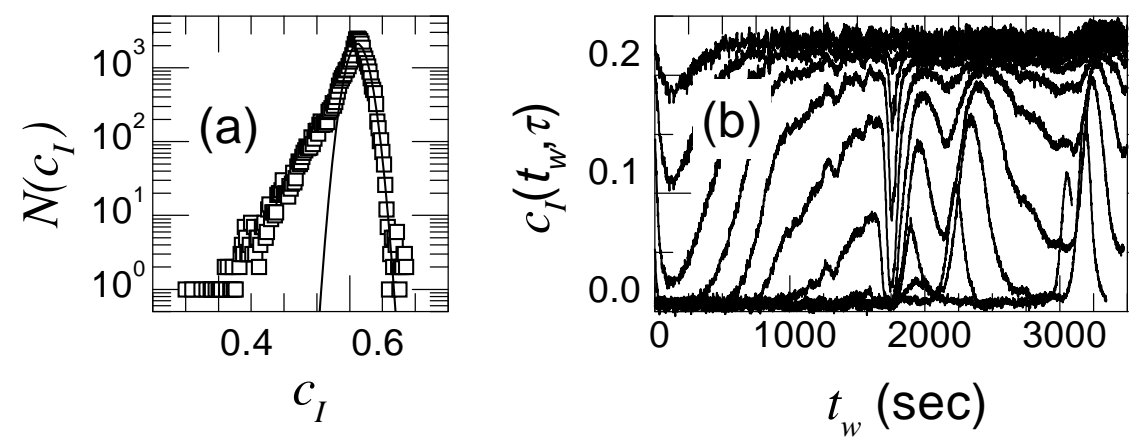

Figure 5. (a): frequency plot of the TRC data for a multilamellar vescicle gel for $\tau=10000 \mathrm{sec}$. The line is a Gaussian obtained by fitting the data to the left of the maximum of $N\left(c_{I}\right)$. (b): TRC data for a concentrated suspension of xenospheres. From top to bottom, $\tau$ varies between 0.5 to $1024 \mathrm{sec}$, doubling from one curve to the next one.

the aging process lead to a substantial loss of correlation that persist over extended periods of time, seemingly indicating that the the gel network is eventually weakened. Interestingly, a similar -albeit temporary- weakening of the gel has also been observed in single scattering experiments on samples at lower volume fractions [8].

To demonstrate the applicability of the TRC method to single scattering experiments, we show in figure 5a the frequency plot $N\left(c_{I}\right)$ for a gel formed by closely packed multilamellar vesicles (details on the sample can be found in [14]). Here, the CCD camera collects the speckle field scattered within a small solid angle centered around a well defined scattering vector $q=10.6 \mu \mathrm{m}^{-1}$; the speckle images are then processed in the same way as described above. In striking analogy with the distributions for the colloidal gels, we find that $N\left(c_{I}\right)$ is strongly skewed to the left indicating temporally heterogeneous dynamics. Similar results are also obtained for a different concentrated surfactant phase, a micellar polycrystal [15] (single scattering geometry, data not shown).

These preliminary results suggest that temporal inhomogeneities are a general feature of the dynamics of jammed materials. Further support to this hypothesis is provided by DWS TRC measurements on another jammed system, a concentrated suspension of poly-vinyl chloride xenospheres, shown in figure $5 \mathrm{~b}$ (the xenospheres are particles a few tens of microns in size composed by permanently aggregated submicrometric primary spheres [16]). As for the other jammed systems discussed above, the dynamics is highly temporally heterogeneous, as demonstrated by the wide variations of $c_{I}$ with $t_{w}$, which are particularly evident for the longest delays $\tau$. Interestingly, we remark that here the dynamical inhomogeneities are rather different from the previous cases, for which the drops of $c_{I}$ are sudden events whose duration is much smaller than the characteristic relaxation time. On the contrary, the much smoother variation of $c_{I}$ observed for the xenospheres indicates that this system slowly explores different dynamical states, characterized by widely different mobility. 
In conclusion, we have introduced a new scheme to explore temporal variations of the degree of correlation, which allows continuous dynamics and temporally heterogeneous or intermittent dynamics to be discriminated. This approach can be used in a wide variety of experimental configurations, both in the single and in the multiple scattering limit, and could be easily extended to X-Photon Correlation Spectroscopy [17, the only requirements being a coherent radiation source and a multielement detector, such as the pixels of a CCD camera. Thanks to the TCR method we have shown that temporal heterogeneities are present in a number of jammed materials, suggesting that intermittency is a general feature of the dynamics of jammed materials. Further work will be needed to fully exploit the information contained in the TRC data, as well for exploring its connection to other techniques, such as higher-order intensity correlation functions [18], which can detect dynamical heterogeneities.

We thank S. Romer for early measurements on the colloidal gels and D. Weitz, L. Ramos and C. Ligoure for useful discussions. Financial support from Région LanguedocRoussillon and the Swiss National Science Foundation (Grant 20-65019.01) is gratefully acknowledged.

\section{References}

[1] For a review, see for example Cipelletti L and Ramos L 2002 Curr Opin Coll Int Sci 7228.

[2] Liu A J and Nagel S D 1998 Nature 39621.

[3] Fielding SM, Sollich P, Cates ME 2000 J Rheol 44323.

[4] Gauckler L J, Graule T and Baader F 1999 Materials Chemistry and Physics 25091.

[5] Romer S, Scheffold F and Schurtenberger P 2000 Phys. Rev. Lett. 854980.

[6] Weitz D A and Pine D J 1993 in Dynamic light scattering Brown W editor (Oxford: Clarendon Press).

[7] Cipelletti L and Weitz D A 1999 Rev. Sci. Instrum. 70, 3214.

[8] Cipelletti L, Manley S, Ball R C and Weitz D A 2000 Phys. Rev. Lett. 842675.

[9] Durian D J, Pine D J and Weitz D A 1991 Science 252686.

[10] Durian D J, Weitz D A and Pine D J 1991, Phys. Rev. A 44 R7902.

[11] Viasnoff V and Lequeux F 2002 Phys. Rev. Lett. 89065701.

[12] Papoulis A 1965 Probability, random variables, and stochastic processes (New York: McGraw-Hill).

[13] Bouchaud J-P and Pitard-Roche E 2001 Eur. Phys. J. E 6231.

[14] Ramos L and Cipelletti L 2001 Phys. Rev. Lett. 87245503.

[15] Cipelletti L, Ramos L, Manley S, Pitard E, Weitz D A, Pashkovski E E and Johansson M, to appear in Faraday Discuss. 123

[16] Encyclopedia of polymer science and engeneering 2nd ed. vol 17 Mark H E et al editors (New York: Wiley-Interscience)

[17] Diat O, Narayanan T, Abernathy D L, Grüber G X 1998 Curr Opin Coll Int Sci 3305.

[18] Lemieux P-A and Durian D J 1999 J. Opt. Soc. Am.A 161651. 